\title{
INFLUÊNCIA DA PRÁTICA DE ATIVIDADE FÍSICA REGULAR NO ÍNDICE DE CAPACIDADE FUNCIONAL SUBMÁXIMA E MOBILIDADE FUNCIONAL DE IDOSAS
}

Elaine Aparecida Lozano da Silva ${ }^{1,5}$, Giovanna Neves Bresqui ${ }^{1,5}$, Andressa Sampaio Pereira ${ }^{2,5}$, Alan José Barbosa Magalhães ${ }^{3,5}$, Vinicius Gustavo Gimenes Turato ${ }^{4,5}$, Juliana Rosini da Silva ${ }^{4,5}$, Cláudia Regina Sgobbi de Faria ${ }^{5}$, Regina Celi Trindade Camargo ${ }^{5}$

Universidade Estadual Paulista - FCT/UNESP. ${ }^{1}$ Especialização em Fisioterapia aplicada a Gerontologia. ${ }^{2}$ Curso de Fisioterapia. ${ }^{3}$ Especialização em Fisioterapia aplicada à Ortopedia e Traumatologia. ${ }^{4}$ Programa de Residência em Saúde, área de Reabilitação Física. ${ }^{5}$ Departamento de Fisioterapia, Presidente Prudente, SP. e-mail: elaine lozano@hotmail.com

\section{RESUMO}

Os idosos tornam-se mais dependente com o avançar da idade, apresentando declínios da capacidade funcional, marcha, equilíbrio e mobilidade. A preservação das mesmas pode mantê-los autônomos e ativos, proporcionando assim um envelhecimento mais saudável. O objetivo foi avaliar a influência da prática regular de atividade física na capacidade funcional submáxima e mobilidade funcional de idosas. Trata-se de um estudo transversal com 39 idosas com idade média de $69 \pm 8,70$ anos. Foram aplicados os testes de caminhada de seis minutos e o Timed Up and Go. Os resultados mostraram que houve aumento significativo entre os resultados da avaliação inicial e final do teste de caminhada de seis minutos, mas em relação ao teste Timed Up and Go, não foi verificada diferença significativa. Dessa forma conclui-se que idosos que praticam atividade física regular apresentam redução e ou manutenção dos níveis de capacidade e mobilidade funcional. Palavras-chave: envelhecimento, atividade física, aptidão física, idosos dependentes, qualidade de vida.

\section{INFLUENCE OF PRACTICE OF PHYSICAL ACTIVITY IN REGULAR INDEX FUNCTIONAL CAPACITY AND MOBILITY FOR THE ELDERLY}

\begin{abstract}
The elderly become more dependent with advancing age, with declines in functional capacity, gait, balance and mobility. The preservation of them can keep them active and autonomous, thus providing a more healthy aging. The objective was to evaluate the influence of regular physical activity in the submaximal functional capacity and functional mobility of elderly. It is a crosssectional study of 39 elderly women with a mean age of $69 \pm 8.70$ years. Tests of six-minute walk and the Timed Up and Go were applied. Results showed a significant increase between the results of the initial evaluation and end of the six-minute walk test, but in relation to the Timed Up and Go test, not significant difference was observed. Thus it is concluded that seniors who engage in regular physical activity have reduced and or maintenance of levels of functional capacity and mobility.
\end{abstract}

Keywords: aging, physical activity, physical fitness, dependent elderly, quality of life. 


\section{INTRODUÇÃO}

Com base em estudos demográficos a população idosa vem se tornando uma realidade em nosso país, e paralelo ao envelhecimento populacional surgem implicações na saúde desta, necessitando de cuidado minucioso e melhor aperfeiçoamento terapêutico em saúde pública, a fim de impelir melhor qualidade de vida à população idosa, reduções do número de hospitalizações e do índice de incapacidades graves ${ }^{1}$.

Todos os seres vivos envelhecem e tornam-se vulneráveis a doenças, principalmente as crônico-degenerativas, que por sua vez podem ser justificadas pelas alterações fisiológicas e bioquímicas que percorrem durante todo o processo do envelhecimento ${ }^{2}$. O envelhecimento se difere de indivíduo para indivíduo, decorrente da existência de diferentes variáveis, como genética, meio ambiente, estilo de vida, hábitos alimentares e socialização, todos podendo interferir negativamente na saúde dos indivíduos quando mal administrados ${ }^{3}$.

Os idosos tendem a se tornar mais dependentes com o avançar da idade, em decorrência dos declínios de capacidade funcional, marcha e mobilidade, tornando-se assim, aspectos de suma importância para a saúde do idoso, pois as preservações das mesmas podem manter o idoso autônomo e ativo, proporcionando assim um envelhecimento mais saudável ${ }^{4,5}$.

A capacidade funcional é definida pela capacidade do individuo em realizar suas atividades de vida diária com potencialidade e sem que haja necessidade de auxilio ${ }^{6}$. As atividades de vida diária englobam atividades de cuidado pessoal tais como tomar banho, vestir-se, levantar-se, caminhar, cozinhar e atividades instrumentais como cozinhar, limpar a casa, fazer compras, uso do telefone e administração de finanças ${ }^{7}$.

$\mathrm{O}$ déficit de marcha, equilíbrio e alterações da mobilidade funcional atenuam o índice de desequilíbrios posturais e quedas do idoso, e podem levar a implicações nas atividades diárias e aumento de hospitalizações do individuo ${ }^{8}$.

A maioria das atividades de vida diária que realizamos no cotidiano, como as citadas acima, estão a nível submáximo, identificando assim importante subsídio para a mensuração do declínio funcional no idoso ${ }^{9}$.

Sendo assim, por meio de uma boa avaliação da capacidade funcional do idoso, podem-se detectar declínios fisiológicos e físicos acentuados, que devem ser reduzidos e ou prevenidos por meio de intervenções, tais como a prática regular de atividade física, que busca minimizar os efeitos deletérios do envelhecimento e proporcionar ao idoso 
melhor qualidade de vida e independência em suas atividades do cotidiano ${ }^{10}$.

O presente trabalho tem como objetivo verificar a influencia da prática regular de atividade supervisionada na capacidade funcional submáxima e mobilidade funcional de idosos ativos.

\section{MÉTODOS}

Foi realizado um estudo transversal com 39 idosas, participantes do programa de fisioterapia preventiva para a terceira idade em Presidente Prudente.

A pesquisa foi encaminhada e aprovada pelo Comitê de Ética em Pesquisa sob 0 protocolo CAAE no 02798312.8.0000.5402 da Faculdade de Ciências e Tecnologia - UNESP, Campus de Presidente Prudente-SP.

\section{Amostra}

As voluntárias foram devidamente informadas sobre os procedimentos e objetivos deste estudo, e após concordarem, assinaram um termo de consentimento livre e esclarecido.

Os critérios de inclusão foram: idade igual ou superior a 60 anos, participar do programa de fisioterapia para a terceira idade citado acima, concordar em assinar o termo de consentimento livre e esclarecido.
Os critérios de exclusão foram: apresentar déficit cognitivo significativo ou alterações que impeçam de realizar o teste.

\section{Programa de atividade física}

O programa regular de atividade física estabelecida, conta com um Protocolo de Exercícios Físicos Supervisionados (PEFS), que é composto por alongamentos, fortalecimento muscular, flexibilidade, coordenação, equilíbrio, memória e relaxamento. As sessões foram realizadas três vezes por semana com uma hora de duração.

\section{Coleta de dados - instrumentos}

Este estudo foi realizado por meio da aplicação do Teste de Caminhada de 6 minutos (TC 6') e o teste Timed Up and Go (TUG). A avaliação inicial foi realizada em março de 2012 e a avaliação final em dezembro do mesmo ano.

a) Teste de Caminhada de 6 minutos - O teste seguiu o protocolo realizado por Britto e Sousa ${ }^{11}$.

O teste foi realizado em um corredor com comprimento mínimo de 30 metros, livre de circulação de pessoas e obstáculos. O avaliador somente caminhou junto ao participante, quando foi observada a necessidade de maior segurança ao mesmo, em situações de déficits de equilíbrio. Nesses 
casos, o avaliador caminhou atrás do participante.

A realização do teste ocorreu por pelo menos duas horas após a última refeição do participante e, este foi instruído a usar roupas e calçados confortáveis, além de manter medicação usual. Antes do inicio do teste, os participantes permaneceram em repouso durante 10 minutos, período onde foram mensurados pressão arterial, oximetria de pulso, nível de frequência cardíaca e respiratória. Durante a realização dos testes, frases de encorajamento foram utilizadas a cada minuto, por meio de frases padronizadas.

Ao término dos testes, os dados vitais coletados inicialmente foram novamente avaliados e calculada a distância percorrida pelo paciente

Para a interpretação dos dados foi utilizada a equação de referência para predição da distância no Teste de Caminhada de 6 minutos segundo Enright e Sherrill ${ }^{12}$.

b) Timed Up and Go

Para a realização do teste foi utilizada uma cadeira sem braços, cronometro e comando verbal. No inicio do teste o paciente foi orientado a se sentar com total apoio de seu dorso no encosto da cadeira e em seguida após o comando "vá" levantar-se sem que haja ajuda dos braços, percorrer o circuito de 3 metros, dar a volta e tornar ao lugar de início sentando-se na cadeira com o total apoio no encosto, o teste foi cronometrado em todo seu percurso ${ }^{13}$.

\section{Análise dos dados}

Para comparação estatística dos resultados das avaliações obtidos no grupo, foi utilizado o Teste KolmogorovSmirnov/Shapiro-Wilk para a verificação da normalidade dos dados. No TC'6, foi utilizado - Teste T-Student para amostras dependentes e dados paramétricos. No TUG foi utilizado o Teste Wilcoxon, para amostras dependentes e dados não-paramétricos. Todos os procedimentos adotaram o valor de significância de $(p=0,05)$. Os cálculos foram realizados com o aplicativo SPSS 17.0 for Windows ${ }^{\circledR}$.

\section{RESULTADOS}

A população deste estudo foi constituída por 39 idosas, com idade média de $69 \pm 8,7$ anos.

Os resultados mostraram que não houve diferença estatisticamente significante entre os resultados da avaliação inicial e final do TC 6' (Tabela 1). Com base nos resultados da avaliação inicial $66,66 \%$ das idosas ultrapassaram os valores preditos de acordo com a equação de Enright e Sherrill e na avaliação final do teste $71,79 \%$ das idosas ultrapassaram o mesmo ${ }^{13}$. 
Tabela 1. Valores da média, desvio padrão e p-valor do teste de caminhada de 6 minutos da avaliação inicial e final.

\begin{tabular}{|l|c|c|c|}
\hline & $\begin{array}{c}\text { Inicial } \\
\text { (Md } \pm \text { DP) }\end{array}$ & $\begin{array}{c}\text { Final } \\
\text { (Md } \pm \text { DP) }\end{array}$ & $p$-valor \\
\hline Pacientes & $493,157 \pm 65,756$ & $493,526 \pm 70,111$ & 0,981 \\
\hline
\end{tabular}

Já no TUG, não houve diferença estatisticamente significante entre 0 resultado da avaliação inicial e final do teste (Tabela 2). Porém na pratica foi observada uma diferença clinica, em $51,41 \%$ das idosas que apresentaram menor tempo de realização do teste quando comparado com a avaliação final.

Tabela 2. Valores da média, desvio padrão e p-valor do Timed Up and Go da avaliação inicial e final.

\begin{tabular}{|l|c|c|c|}
\hline & $\begin{array}{c}\text { Inicial } \\
\text { (Med } \pm \text { IQ) }\end{array}$ & $\begin{array}{c}\text { Final } \\
\text { (Med } \pm \text { IQ) }\end{array}$ & $p$-valor \\
\hline Pacientes & $8,000 \pm 2,50$ & $8,250 \pm 3,94$ & 0,717 \\
\hline
\end{tabular}

\section{DISCUSSÃO}

Como observado no presente estudo, às idosas apresentaram valores próximos quando se referido a distância percorrida nos dois testes de caminhada de 6 minutos realizados, sendo 493,15 metros para avaliação inicial e 493,52 metros para avaliação final, diferenças essas não significativas, porém á distância predita na avaliação inicial foi 451,11 metros e na avaliação final 434,09 metros.

No estudo realizado com 17 idosas com diagnóstico de osteoporose, ocorreu aumento significativo da distância percorrida no TC 6 minutos após três meses de intervenção, esse aumento pode estar relacionado com os exercícios de fortalecimento de membros inferiores ${ }^{14}$.

Segundo o estudo realizado com 74 idosas, sendo que dessas 37 não realizaram atividade física regular e outras 37 participaram de duas aulas semanais de hidroginástica no período de três meses, constataram aumento da distancia percorrida no TC 6 minutos, dados esses enfatizam que - exercício físico aumenta a capacidade aeróbica de 10 a $40 \%$ no idoso ativo ${ }^{15}$.

Apesar da amostra do presente estudoser pequena e também não apresentar diferenças significativas quando comparado os dados do TUG. No estudo realizado com 18 idosas com idade média de 
69 anos, as idosas do grupo de intervenção foram submetidas ao protocolo de tratamento constituído por exercícios de equilíbrio, alongamentos e fortalecimento, constatou-se que idosas praticantes de atividade física apresentam melhor mobilidade, equilíbrio e agilidade quando comparado com idosas sedentário, dado estes identificados por meio do teste TUG ${ }^{16}$.

$$
\text { O estudo realizado por Pedrosa e }
$$
Holanda $^{17}$ corroboram com os dados encontrados no presente estudo, resultando que idosos que caminham por maior tempo no TUG, percorrem uma menor distancia no TC 6', dados este também encontrado em nosso estudo. Idosos que apresentam maior índice de redução da mobilidade funcional se tornam mais dependentes do auxilio de terceiros para realizar as atividades do cotidiano, alem de contribuírem para implicações das funções motoras e cardiovasculares.

Em ambos os testes quando comparado com os resultados iniciais e finais individualmente pode-se constatar 0 aumento da distância percorrida no teste de caminhada de 6 minutos e redução do tempo no teste TUG em grande parte dos participantes, dados esses importantes clinicamente.

A participação de programa de atividade físico regular é benéfica quando diz respeito às alterações funcionais do envelhecimento, proporcionando assim redução, manutenção e prevenção das mesmas ${ }^{18}$. Os idosos se tornam vulnerável ao risco de quedas e lesões durante as atividades de vida diária, no entanto quando o idoso abandona o habito de sedentarismo, esses riscos apresentam menores índices de ocorrência ${ }^{19}$.

\section{CONCLUSÃO}

Conclui-se que as idosas participantes de atividade física regular não apresentaram reduções significantes estatisticamente a níveis de capacidade e mobilidade funcional, porém clinicamente apresentaram melhoria dos mesmos.

\section{REFERÊNCIA}

1. NUNES DP, NAKATANI AYK, SILVEIRA EA, BACHION MM,SOUZA MR. Capacidade Funcional, condições socioecônomicas e de saúde de idosos atendidos por equipes de Saúde da família de Goiana (GO,Brasil). Ciência \& Saúde Coletiva. 2010; 15 (6): 28872898.

\section{FERREIRA OGL, MACIEL SC, COSTA} SMG, SILVA AO, MOREIRA MASP.Envelhecimento Ativo e sua relação com a Independência Funcional. Texto Contexto Enferm. 2012; 21(3): 513-8.

3. FECHINE BRA, TROMPIERI N. O processo de envelhecimento: As principais alterações que acontecem com o idoso com o passar dos anos. Rev. Interscienceplace.2012; 1(7).

4. SOUZA IP, SANTOS LM, SANTANA VS, FEITOSA AG. Capacidade Funcional de idosos com doença de Alzheimer e Parkinson. Revista Pesquisa em Fisioterapia. 2014; $4(1): 78-84$. 
5. MACIEL ACC, GUERRA RO. Fatores associados à alterações da mobilidade em idosos residentes na comunidade. Rev. bras. Fisioter.2005; 9(1):17-23.

6. OLIVEIRA PH, MATTOS IE. Prevalência e fatores associados à incapacidade funcional em idosos institucionalizados no Município de Cuiabá, Estado de Mato Grosso, Brasil, 2009-2010. Rev. Epidemiol. Serv. Saúde. 2012; 21(3): 395-406.

7. FRANCHI KMB, MONTEIRO LZ, ALMEIDA SB, PINHEIRO MHNP, MEDEIROS AIA, MONTENEGRO RM, JÚNIOR RMM, et al. Capacidade Funcional e Atividade Física de Idosos com Diabetes Tipo 2. Revista Brasileira de Atividade Física \& Saúde. 2009; 13(3).

\section{GONÇALVES DFF, RICCI NA, COIMBRA}

AMV. Equilíbrio funcional de idosos da comunidade: comparação em relação ao histórico de quedas.RevBrasFisioter.2009;13(4):316-23.

9. BARATA VF, GASTALDI AC, MAYER AF,SOLOGUREN MJJ.Avaliação das equações de referência para predição da distância percorrida no teste de caminhada de seis minutos em idosos saudáveis brasileiros. Rev. bras. fisioter. 2005; 9 (2):165-171.

10. GONÇALVES, M. Contribuições da fisioterapia/exercício físico para pacientes idosos atendidos na Estratégia Saúde da Família (ESF). Ensaios e Ciência: Ciências Biológicas, Agrárias e da Saúde.2011; 15 (1): 243-260.

11. BRITTO RR, SOUSA LAP. Teste de caminhada de seis minutos uma normatização brasileira. Fisioterapia em Movimento. 2006; 19 (4): 49-54.

12. ENRIGHT PL,SHERRILL DL. Reference equations for the six-minute walk in healthy adults.Am. J. Resp. Crit. Care

Med.1998;1384-1387.
13. ROGERS ME, ROGERS NL, TAKESHIMA $\mathrm{N}$, ISLAM MM. Methods to assess and improve the physical parameters associated with fall risk in older adults. Prev Med., v.36, n.3, p.255-64, 2003.

14. NAVEGA MT, AVEIRO MC,OISHI J. Alongamento, caminhada e fortalecimento dosMúsculos da coxa: um programa de atividadeFísica para mulheres com osteoporose. Rev. bras. fisioter. 2003;7 (3): 261-267.

15. ALVES RV, MOTA J,COSTA MC, ALVES JGB.Aptidão física relacionada à saúde de idosos: influência da hidroginástica. RevBrasMed Esporte. 2004; 10(1).

16. SILVA AM, SILVA RB, GUERRA RA, SIQUEIRA, GR. Fisioterapia em relação à marcha e ao equilíbrio em idosos. RBPS. 2011; 24(3): 207-213.

17. PEDROSA R, HOLANDAG.Correlação entre os testes da caminhada, marcha estacionária e TUG em hipertensas idosas. Rev. bras. Fisioter.2009;13(3).

18. RIBEIRO F, GOMES S,TEIXEIRA F,BROCHADO G,OLIVEIRA J. Impacto da prática regular de exercício físico no equilíbrio, mobilidade funcional e risco de queda em idosos institucionalizados. Rev. Port. Cien. Desp. 2009; 9(1): 36-42.

19. COUTO FCO, BASTOS IRS, DIAS VB, CAMPOS CE.Comparação da agilidade de mulheres idosas ativas e sedentárias.Coleção Pesquisa em Educação Física.2010;9(5).

Recebido para publicação em 12/08/2014 Revisado em 29/08/2014

Aceito em 12/09/2014 